\section{AB0364 EFFICACY OF BARICITINIB (BARI) IN PATIENTS WITH RHEUMATOID ARTHRITIS (RA) WHOSE RESPONSE WAS INADEQUATE TO TOFACITINIB (TOFA)}

T. Yamane ${ }^{1}$, A. Hashiramoto ${ }^{2}{ }^{1}$ Kakogawa Central City Hospital, Kakogawa, Japan; ${ }^{2}$ Kobe University Graduate School of Health Sciences, Kobe, Japan

Background: Currently, four types of JAK inhibitors are approved for the treatment of RA in Japan, however, they often show differences in clinical efficacies presumably due to their JAK selectivity.

Objectives: To investigate the efficacy of Bari for patients with Tofa-inadequate response (IR), clinical profiles of seven Tofa IR patients were evaluated.

Methods: We performed a single-center retrospective study on seven Tofa IR patients (female:7) who were switched to Bari. Items evaluated were as follows; patient's baseline characteristics, continuation rate of Bari, swollen joint count, tender joint count, C-reactive protein (CRP), matrix proteinase 3 (MMP3), physician's and patient's visual analog scale (VAS), disease activity assessed by Disease Assessment Score of 28 joints - C-reactive protein (DAS 28-CRP), simplified disease activity index (SDAl) and clinical disease activity index (CDAI) at 2, 4, 8, 12 weeks, and the dosage of prednisone (PSL).

Results: Patient's mean age was 56.4 and mean disease duration was 9.2 years. Tofa had administered for 3.4 months (range 1-10) before switching to Bari. All patients were positive for both anti-citrullinated protein antibodies and rheumatoid factor. The number of prior biologics use was 2.3 (range1-4). 3 patients had concomitant MTX use (mean dosage $5.3 \mathrm{mg} /$ week) and 4 had prednisone (mean dosage $3.3 \mathrm{mg} / \mathrm{day}$ ). Mean swollen joint counts and tender joint counts were 5.0 (range 1-15) and 5.3 (range 1-15), respectively. Physician's and Patient's VAS 0-100 were $71 \mathrm{~mm}$ (range 50-90) and $73 \mathrm{~mm}$ (range 50-80), mean CRP levels were $1.6 \mathrm{mg} / \mathrm{dl}$ (range 0.01-4.3) and MMP3 levels were $357.3 \mathrm{mg} / \mathrm{dl}$ (range 27-933). Mean SDAI, CDAI and DAS-28-CRP were 26.3 (range17-50), 24.7 (range 17-47) and 4.5, respectively. When enrolled, 4 of 7 patients corresponded to moderate disease activity (MDA) and 3 were in high disease activity (HDA) by all composite measures. After switched to Bari, the patient's VAS significantly decreased at week 2 ( $P<0.01)$. CDAI, SDAI and DAS28-CRP also showed a significant improvement at week $4,(P<0.05)$. At week 4, 2 patients discontinued Bari due to the lack of efficacy or acute exacerbation of interstitial pneumonia. Thus, continuation rate of Bari at week 12 was $71.4 \%$. Among 5 patients who continues Bari, all patients achieved low disease activity (LDA) by SDAI and CDAl at week 12. By DAS 28-CRP, 2 patients achieved MDA, 2 achieved LDA and 1 achieved remission (mean 2.6), respectively, at week 12,3 of 4 patients taking PSL were able to withdraw that before week 12 (Figure).

Conclusion: Result showed the efficacy of Bari for patients with Tofa IR, representing inadequate improvements of patient's VAS with Tofa. The efficacy of Bari can be expected from the early stage and continued up to 12 weeks after switching. This difference in therapeutic effect may be due to each mechanism of action, Bari inhibits JAK1/2, whereas Tofa mainly inhibits JAK1 /3 (1). JAK2 is important for signal by GM-CSF, which has shown potential as an important therapeutic target in RA (2).Inhibition of JAK2 and GM-CSF may be the reason for Bari efficacy in Tofa IR patients.

References:

[1] O'Shea JJ et al. Back to the future: oral targeted therapy for RA and other autoimmune diseases. Nat Rev Rheumatol 2013 Mar;9(3):173-82.

[2] Wicks IP et al. Targeting GM-CSF in inflammatory diseases. Nat Rev Rheumatol. 2016 Jan;12(1):37-48.
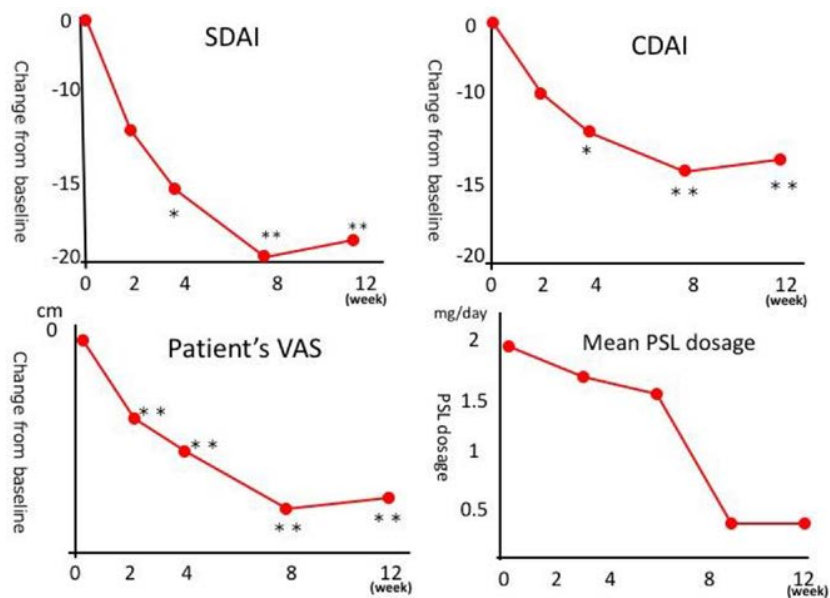

Duration from switching Tofa to Bari
Disclosure of Interests: None declared DOI: 10.1136/annrheumdis-2020-eular.4349

\section{AB0365 COMPARISON OF SUSTAINED CLINICAL REMISSION AND/OR LOW DISEASE ACTIVITY RATE BETWEEN RAPIDLY AND GRADUALLY DE-ESCALATION OF BARICITINIB IN RHEUMATOID ARTHRITIS.}

M. Yamasaki ${ }^{1}{ }^{1}$ Shin-Yokohama Arthritis and Rheumatology Clinic, Yokohama, Japan

Background: However baricitinib, an oral selective inhibitor of Janus kinase (JAK) 1 and 2, improved signs and symptoms of rheumatoid arthritis (RA), it is unknown who can taper or stop baricitinib and strategies for de-escalation.

Objectives: We analyze predictors of tapering of withdrawal failure in rheumatoid arthritis (RA) patients treated with baricitinib. This study will assess and compare (1) characteristic of patients who achieve remission (REM) or low disease activity (LDA) as who can taper baricitinib and (2) two de-escalation methods, rapidly and gradually de-escalation in patients who respond first-line therapy. Methods: Cases were recruited to SHin-yokohama Arthritis REgister (SHARE) between 2015 and 2019 ( $n=3,674)$. Patients were diagnosed according to ACR/ EULAR 2010 classification criteria, and treated with DMARDs which included baricitinib 2mg/day ( $\mathrm{n}=154)$. In 154 cases, Clinical Disease Activity Index (CDAl), Health Assessment Questionnaire-Disability Index (HAQ-DI), anti-CCP2 and patients clinical parameters were analyzed. Two de-escalation methods were compared in this study. In rapidly de-escalation methods, baricitinib were stopped in patients with stable REM/LDA over 12 weeks. In gradually de-escalation methods, baricitinib were decreased to $50 \%, 42 \%, 28 \%, 14 \%$ in order with stable REM/LDA over 12 weeks.

Results: In 154 (Male25, Female129 cases, RA duration 11.4+/-8.0 years) cases, CDAl at baricitinib-start was $20.6+/-12.4$ and titer of anti-CCP2 was $242.6+/$ $516.5 \mathrm{U} / \mathrm{ml}$. 126 cases $(81.8 \%)$ were more than 2 years of RA duration and 49 cases $(31.8 \%)$ had persistency of signs and/or symptoms suggestive of inflammatory RA disease activitiy, despite prior treatment with csDMARDs and at least two biologic DMARDs. 33 cases (21.4\%) were biologic DMARDs naive.

(1)"Multivariate logistic regression examined the predictors to detect who can taper baricitinib" However there were no differences in duration of RA, onset age of RA, biologics and/or JAK inhibitors naïve, anti-CCP2 titer and CDAl at the start baricitinib, patients who showed decrease of CDAl at 12 weeks were correlated with achievement of remission (REM) or low disease activity (LDA) in patients treated with baricitinib (OR $0.964,95 \% \mathrm{Cl} 0.934-0.996, \mathrm{p}=0.010)$. ROC analysis of $\triangle \mathrm{CDAl}$ at 12 weeks is cut-off value of $-6.6(\mathrm{p}=0.011)$.

(2)"Comparison of sustained REM and/or LDA rate between rapidly and gradually de-escalation of baricitinib in rheumatoid arthritis" 11 cases were tapered baricitinib with rapidly de-escalation methods and 60 patients were with gradually de-escalation. Mean times to start taper baricitinib in rapidly and gradually de-escalation group were $4.6+/-1.6$ months and $5.9+/-2.2$ months respectively. Gradually de-escalation methods showed less relapse rate compared with rapidly de-escalation after tapered baricitinib for 6 months ( $18.3 \%$ vs. $54.5 \%$, $\mathrm{p}=0.018$ ). There were no differences in clinical features such as anti-CCP2, $\mathrm{CDAl}$ and administration period of baricitinib between non-relapse and relapse patients in gradually escalation methods.

Conclusion: A combination of $\triangle \mathrm{CDAl}$ at 12 weeks and tapering baricitinib using gradually de-escalation methods may help to predict successful baricitinib deduction in RA patients with sustained clinical REM and/or LDA.

References:

[1] Ann Rheum Dis. 2019;78:171

[2] Rheumatology. 2019;58:110

[3] An Rheum Dis. 2015;74:19

Disclosure of Interests: None declared

DOI: 10.1136/annrheumdis-2020-eular.3991

\section{SLE, Sjögren's and APS - treatment}

\section{AB0366 \\ DIFFUSE ALVEOLAR HEMORRHAGE IN LUPUS NEPHRITIS PATIENTS: MULTICENTER RECTROSPECTIVE STUDY}

S. Abdulaziz ${ }^{1}$, H. Halabi ${ }^{2}$, S. Bahlas ${ }^{3}$, S. Attar ${ }^{3}$, M. Dessougi ${ }^{4}$, A. Alhouri ${ }^{5}$, A. Taruti ${ }^{6}$, H. Alrayes ${ }^{7}$, A. H. A. Albeity ${ }^{8}{ }^{1}$ King Fahd Hospital, Jeddah, Saudi Arabia; ${ }^{2}$ King Faisal Specialist Hospital, Jeddah, Saudi Arabia; ${ }^{3}$ King Abdulaziz University, Jeddah, Saudi Arabia; ${ }^{4}$ Armed Forces Hospital, Riyadh, Saudi Arabia; ${ }^{5}$ Qatif Central Hospital, Damman, Saudi Arabia; ${ }^{6}$ Qatif Central Hospital, Jeddah, Saudi Arabia; ${ }^{7}$ King Sultan Military Hospital, Riyadh, Saudi Arabia; ${ }^{8}$ King Faisal Specialist Hospital, Jeddah, Saudi Arabia 